\title{
On the applicability of the spherical wave expansion with a single origin for near-field acoustical holography
}

Gomes, J.; Hald, J.; Juhl, P.; Jacobsen, Finn

Published in:

Journal of the Acoustical Society of America

Link to article, DOI:

$10.1121 / 1.3068451$

Publication date:

2009

Document Version

Publisher's PDF, also known as Version of record

Link back to DTU Orbit

Citation (APA):

Gomes, J., Hald, J., Juhl, P., \& Jacobsen, F. (2009). On the applicability of the spherical wave expansion with a single origin for near-field acoustical holography. Journal of the Acoustical Society of America, 125(3), 15291537. https://doi.org/10.1121/1.3068451

\section{General rights}

Copyright and moral rights for the publications made accessible in the public portal are retained by the authors and/or other copyright owners and it is a condition of accessing publications that users recognise and abide by the legal requirements associated with these rights.

- Users may download and print one copy of any publication from the public portal for the purpose of private study or research.

- You may not further distribute the material or use it for any profit-making activity or commercial gain

- You may freely distribute the URL identifying the publication in the public portal 


\title{
On the applicability of the spherical wave expansion with a single origin for near-field acoustical holography
}

\author{
Jesper Gomes ${ }^{a)}$ \\ Institute of Sensors, Signals and Electrotechnics, University of Southern Denmark, Campusvej 55, \\ DK-5230 Odense M, Denmark \\ Jørgen Hald \\ Brüel \& Kjar Sound and Vibration Measurement A/S, Skodsborgvej 307, DK-2850 Narum, Denmark \\ Peter Juhl \\ Institute of Sensors, Signals and Electrotechnics, University of Southern Denmark, Campusvej 55, \\ DK-5230 Odense M, Denmark
}

\begin{abstract}
Finn Jacobsen
Acoustic Technology, Department of Electrical Engineering, Technical University of Denmark, Building 352, Ørsteds Plads, DK-2800 Kgs. Lyngby, Denmark
\end{abstract}

(Received 29 August 2008; revised 20 November 2008; accepted 12 December 2008)

\begin{abstract}
The spherical wave expansion with a single origin is sometimes used in connection with near-field acoustical holography to determine the sound field on the surface of a source. The radiated field is approximated by a truncated expansion, and the expansion coefficients are determined by matching the sound field model to the measured pressure close to the source. This problem is ill posed, and therefore regularization is required. The present paper investigates the consequence of using only the expansion truncation as regularization approach and compares it with results obtained when additional regularization (the truncated singular value decomposition) is introduced. Important differences between applying the method when using a microphone array surrounding the source completely and an array covering only a part of the source are described. Another relevant issue is the scaling of the wave functions. It is shown that it is important for the additional regularization to work properly that the wave functions are scaled in such a way that their magnitude on the measurement surface decreases with the order. Finally, the method is applied on nonspherical sources using a vibrating plate in both simulations and an experiment, and the performance is compared with the equivalent source method.
\end{abstract}

(C) 2009 Acoustical Society of America. [DOI: 10.1121/1.3068451]

PACS number(s): 43.60.Sx, 43.60.Pt, 43.20.Rz [EGW]

Pages: $1529-1537$

\section{INTRODUCTION}

Near-field acoustical holography ${ }^{1}(\mathrm{NAH})$ is a technique that reconstructs the sound pressure, particle velocity, and/or sound intensity on the surface of a sound source based on the acoustic field measured at a set of positions close to the source. Knowing precisely the areas with highest noise radiation is an important prerequisite for choosing an effective noise reduction strategy.

One approach within a variety of NAH methods makes use of the spherical wave expansion with a single origin. The idea is to express the sound field as the truncated wave expansion, and then estimate the coefficients of the included expansion functions. Assuming that the truncated series is an acceptable approximation to the true field, the calculated coefficients are reused to estimate the sound field at positions different from the measurement positions, e.g., on the source. This type of approach can be divided into two subcategories.

\footnotetext{
a) Author to whom correspondence should be addressed. Electronic mail: jgomes@bksv.com. Also at Brüel \& Kjær Sound and Vibration, Skodsborgvej 307, DK-2850 Nærum, Denmark.
}

The first subcategory exploits the fact that the expansion functions are orthogonal on a spherical surface, and by sampling the sound field with a spherical microphone array the coefficients can be estimated using numerical integration. ${ }^{2}$ The second subcategory finds a set of coefficients to the truncated expansion by fitting the model to the measured data, e.g., by minimization of the difference between the measured sound pressure and the predicted sound pressure at the microphone positions in a least squares sense. ${ }^{3-7}$ The latter approach does not rely on the orthogonality of the wave functions, and hence the array does not need to be of spherical shape. The present paper focuses only on this approach.

The truncation is required for practical reasons since the expansion has an infinite number of terms, but it also benefits the reconstruction accuracy because of its inherent regularizing effect. The strong radial decay of the high order terms makes the problem more ill posed, which means that the truncation of the expansion makes the method less sensitive to noise in the measured data. Other ways of regularizing the problem is by use of Tikhonov regularization or truncated singular value decomposition (TSVD) ${ }^{8}$ Often, the truncation of the spherical waves is used as the only regularization 
method. In that case, the SVD, which is required in the TSVD and normally also for Tikhonov regularization, is avoided, which saves computation time. This paper investigates how well the truncation of the spherical wave expansion works as a regularization method, and determines the circumstances where other regularization approaches such as Tikhonov or the TSVD become necessary.

Research within NAH based on the single origin spherical wave expansion (SOSWE) often uses the wave expansion directly as it appears from the mathematical derivation without scaling or normalizing the spherical Hankel functions. ${ }^{3-7}$ In Ref. 9 the Hankel functions are normalized on the measurement surface, but without any arguments given for this choice. To the authors' knowledge there are no published studies on the consequence of not scaling the functions. The present paper demonstrates the importance of the scaling and suggests scaling the waves on the surface of the source.

In principle the SOSWE approach has problems with mapping on irregular source surfaces. For example, for exterior problems the spherical wave model is valid only outside the minimum sphere (the smallest sphere that encloses the source with its center at the origin of the spherical expansion). This means that all measurement and calculation points should be outside the minimum sphere, which is possible only if the source surface to be mapped has a spherical shape. However, results have shown that an acceptable accuracy can be achieved even when dealing with nonspherical source surfaces. ${ }^{3,5,7}$

The equivalent source method $(\mathrm{ESM})^{10-12}$ will be used as a benchmark method when investigating the performance of the SOSWE approach on nonspherical sources. ESM approximates the sound field by a superposition of the sound fields of simple sources distributed inside the source, and it does not require the source to be of separable geometry.

\section{OUTLINE OF THEORY}

\section{A. Single origin spherical wave expansion}

In this paper only exterior (free-field) problems are considered, and hence the expansion only includes outgoing spherical waves. At a point outside the minimum sphere, $\mathbf{r}$ $=(r, \theta, \phi)$, where $\theta$ and $\phi$ are the zenith and azimuth angle, respectively, the sound pressure can then be expressed as

$$
p(\mathbf{r})=\sum_{n=0}^{\infty} \sum_{m=-n}^{n} \widetilde{a}_{n m} h_{n}(k r) Y_{n}^{m}(\theta, \phi),
$$

where $h_{n}$ is the $n$th order spherical Hankel function of the second kind (with the $e^{i \omega t}$ sign convention), $k$ is the wavenumber, $\widetilde{a}_{n m}$ are the unknown expansion coefficients, and $Y_{n}^{m}$ is a "spherical harmonic."

In the SOSWE method the sound field is approximated as a truncated version of Eq. (1),

$$
p(\mathbf{r}) \approx \sum_{n=0}^{N} \sum_{m=-n}^{n} \tilde{a}_{n m} h_{n}(k r) Y_{n}^{m}(\theta, \phi)=\sum_{j=1}^{J} \tilde{a}_{j} \widetilde{\Psi}_{j}(\mathbf{r}),
$$

where $\widetilde{a}_{j}=\widetilde{a}_{n m}$ and $\widetilde{\Psi}_{j}(\mathbf{r})=h_{n}(k r) Y_{n}^{m}(\theta, \phi)$ are elementary wave functions with $j \in\left\{n^{2}+n+m+1\right\}=\left\{1,2, \ldots,(N+1)^{2}\right\}$. The method assumes that the consequence of truncating the expansion at some number, $J$, is acceptable. At some limit the high order elementary waves cannot be sampled adequately with a given microphone spacing, which makes it reasonable to truncate the series. Also, the high order Hankel functions have a strong decay in the region between the reconstruction surface and the measurement surface, which causes potentially large errors during the reconstruction process if the measurement noise contains high order components, i.e., the truncation has a regularizing effect.

The magnitude of the radial part of the elementary wave functions in Eq. (2) increases with the order $n$ for a given distance from the expansion origin. As will be demonstrated in Sec. III A, the SOSWE method in general performs better if the elementary wave functions are scaled so that their magnitude decreases on the measurement surface with the order. This can be done by rewriting Eq. (2) as

$$
p(\mathbf{r}) \approx \sum_{n=0}^{N} \sum_{m=-n}^{n} a_{n m} \frac{h_{n}(k r)}{h_{n}\left(k r_{s}\right)} Y_{n}^{m}(\theta, \phi)=\sum_{j=1}^{J} a_{j} \Psi_{j}(\mathbf{r}),
$$

where $\quad a_{j}=a_{n m}=\widetilde{a}_{n m} h_{n}\left(k r_{s}\right) \quad$ and $\quad \Psi_{j}(\mathbf{r})$ $=\left[h_{n}(k r) / h_{n}\left(k r_{s}\right)\right] Y_{n}^{m}(\theta, \phi)$ defines a new set of elementary wave functions. The radial distance, $r_{s}$, defines the surface of a sphere at which the radial part of the functions has equal amplitude. There are in principle no restrictions on the position of the scaling surface. If it is placed between the expansion origin and the source surface, then the elementary wave functions corresponding to a high order, $n$, have the lowest magnitude on both the source and measurement surface, and if it coincides with the source surface the magnitude is roughly the same on this surface for all waves (assuming a spherical source shape), whereas it decreases with $n$ on the measurement surface.

Equation (3) can be used to express the sound pressure at $M$ field positions, and on matrix form this yields

$$
\mathbf{p}=\mathbf{B a},
$$

where $[\mathbf{a}]^{J \times 1}$ contains the coefficients, $a_{j}$, [ $\left.\mathbf{p}\right]^{M \times 1}$ contains the sound pressure at $M$ field points, and the elements of $[\mathbf{B}]^{M \times J}$ are the values of the $j$ th elementary wave at the $m$ th field point.

If $\mathbf{p}$ contains measured pressure data, then the expansion coefficients are the only unknowns in Eq. (4). The number of waves and the number of measurement points are not necessarily equal, yielding an overdetermined or underdetermined system of equations. To get a unique solution the problem is solved using least squares and least norm for $M \geq J$ and $M$ $<J$, respectively,

$$
\mathbf{a}= \begin{cases}\left(\mathbf{B}^{H} \mathbf{B}\right)^{-1} \mathbf{B}^{H} \mathbf{p} & \text { for } M \geq J \\ \mathbf{B}^{H}\left(\mathbf{B B}^{H}\right)^{-1} \mathbf{p} & \text { for } M<J,\end{cases}
$$

where ${ }^{H}$ denotes the Hermitian transpose.

The computation of the SVD yields $\mathbf{B}=\sum_{i=1}^{I} \mathbf{u}_{i} \sigma_{i} \mathbf{v}_{i}^{H}$, where $\mathbf{u}_{i}$ and $\mathbf{v}_{i}$ are the left and right singular vectors, respectively, and $\sigma_{i}$ are the singular values. If only the terms corresponding to nonzero singular values are included, i.e., $I$ $=\operatorname{rank}(\mathbf{B})$, Eq. (5) can then be written as 


$$
\mathbf{a}=\sum_{i=1}^{I} \frac{\mathbf{u}_{i}^{H} \mathbf{p}}{\sigma_{i}} \mathbf{v}_{i}
$$

for both the overdetermined and underdetermined cases. If the condition number of $\mathbf{B}$ is high, additional regularization is needed in order to prevent strong amplifications of the measurement noise in $\mathbf{p}$. This can be done using the TSVD to get a regularized solution vector

$$
\mathbf{a}_{\mathrm{reg}}=\sum_{i=1}^{I_{\mathrm{reg}}} \frac{\mathbf{u}_{i}^{H} \mathbf{p}}{\sigma_{i}} \mathbf{v}_{i}
$$

The truncation parameter, $I_{\text {reg }}$, can be determined using automated parameter choice methods such as the L-curve analysis or generalized cross-validation (GCV). ${ }^{8}$

As mentioned earlier, the truncation of the wave expansion is often used as the only type of regularization, which means that the expansion coefficients can be found directly from Eq. (5). The computation time increases if the TSVD is introduced in addition, but as demonstrated in Sec. III C, in many cases it will give a more accurate reconstruction result.

After the calculation of $\mathbf{a}_{\text {reg }}$, these coefficients can be reused in Eq. (3) to calculate the pressure on the source surface, and the particle velocity on the surface of the source can be obtained by use of Euler's equation. ${ }^{1}$

\section{B. Equivalent source method}

ESM will be used for benchmarking in this paper. The principle behind the method is quite similar to that of SOSWE, but it is based on a distributed set of equivalent sources, i.e., multiple origins, and the method normally only uses the monopoles from the wave expansion. The sound field in a point $\mathbf{r}$ is estimated as

$$
p(\mathbf{r}) \approx \sum_{j=1}^{J} a_{j} G\left(\mathbf{r}, \mathbf{r}_{j}\right),
$$

where $G\left(\mathbf{r}, \mathbf{r}_{j}\right)$ is the free-space Green's function with its origin at a point $\mathbf{r}_{j}$. The pressure at a set of field points can then be expressed in the same form as for SOSWE in Eq. (4), and the coefficients can be found by use of, for instance, Eq. (7). Finally, the resulting coefficients of the equivalent sources, $\mathbf{a}_{\text {reg }}$, can be used to estimate the pressure and/or particle velocity on the source surface.

\section{SIMULATED MEASUREMENTS}

In all the test cases that follow, only the prediction of the particle velocity is considered, and the resulting reconstruction error is calculated as

$$
\frac{\|\widetilde{\mathbf{v}}-\mathbf{v}\|_{2}}{\|\widetilde{\mathbf{v}}\|_{2}} \cdot 100 \%
$$

where $\widetilde{\mathbf{v}}$ is a vector of the "true" velocities at a set of reconstruction points, and $\mathbf{v}$ contains the estimated velocities. Imperfections in the setup are simulated by introducing normal distributed random amplitude and phase mismatch between the microphones with standard deviations of $0.2 \mathrm{~dB}$ and $1^{\circ}$, respectively. The resulting signal-to-noise ratio (SNR) was

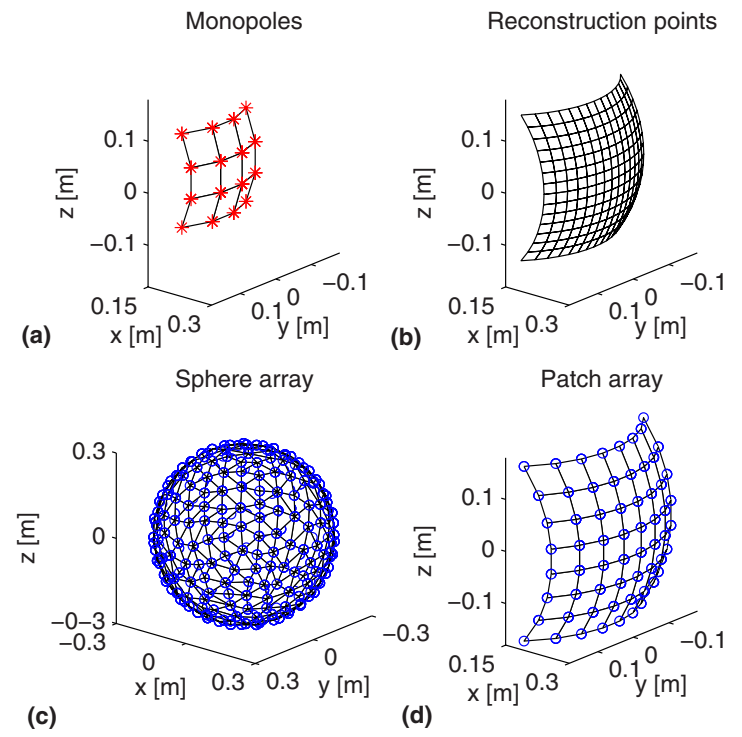

FIG. 1. (Color online) Simulated test case with (a) $4 \times 4$ monopoles located on a patch of a virtual spherical surface with a radius of $0.2 \mathrm{~m}$, (b) 15 $\times 15$ reconstruction points on a patch of a another spherical surface with a radius of $0.25 \mathrm{~m}$, (c) an array of 303 measurement positions located on sphere with a radius of $r_{m}=0.3 \mathrm{~m}$, and (d) an $8 \times 8$ array with all measurement points located at $r_{m}=0.3 \mathrm{~m}$.

approximately $30 \mathrm{~dB}$ in all the simulated results. Tests have also been made with random background noise with the same SNR, but this did not have any effect on the overall conclusions. Hence, only the results based on mismatch errors are shown in the present paper.

The first test case to be considered consists of monopoles that are distributed on a patch of a virtual spherical surface with a radius of $0.2 \mathrm{~m}$, as shown in Fig. 1(a). Their positions correspond to a regular grid of $4 \times 4$ points in the $y z$-direction with a spacing of $6 \mathrm{~cm}$, which is projected onto the virtual sphere. The phase difference between neighboring monopoles is set to $180^{\circ}$ to create an evanescent sound field. The reconstruction points in Fig. 1(b) correspond to 15 $\times 15$ points with a spacing of $2 \mathrm{~cm}$ in the $y z$-direction, which are projected onto a spherical surface of radius $r_{s}=0.25 \mathrm{~m}$. Two different simulated arrays will be considered. In Fig. 1(c) there are 303 measurement positions placed on a spherical surface with a radius of $r_{m}=0.3 \mathrm{~m}$ with an average spacing of $5 \mathrm{~cm}$ between the measurement positions. The patch array in Fig. 1(d) is the projection of every other of the 15 $\times 15$ reconstruction points [see Fig. 1(b)] onto the spherical surface with a radius of $r_{m}=0.3 \mathrm{~m}$, yielding $8 \times 8$ points covering an area slightly larger than the reconstruction surface. The origin of the spherical wave expansion will be placed at $\mathbf{r}=(0,0,0) \mathrm{m}$.

\section{A. Scaling the wave functions}

The influence of the scaling on the reconstruction accuracy is investigated using the simulated setup just mentioned. However, only the sphere array in Fig. 1(c) will be considered in this section.

The SVD of the matrix, $\mathbf{B}$, gives valuable insight into the problem to be solved, and Fig. 2 shows the first and last three left singular vectors with and without the scaling of the 

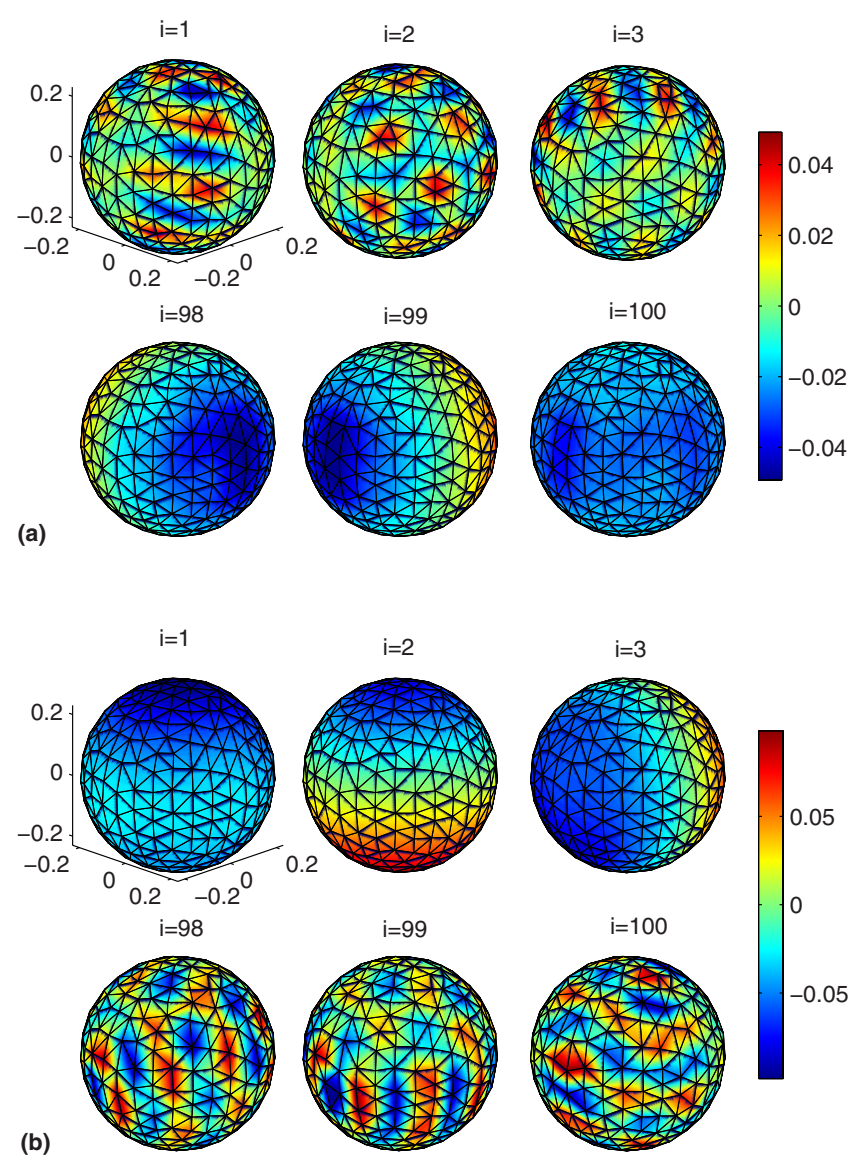

FIG. 2. (Color online) Examples of real part of the left singular vectors, $\mathbf{u}_{i}$, plotted on the spherical measurement surface with radius, $r_{m}=0.3 \mathrm{~m}$. The frequency is $f=500 \mathrm{~Hz}$, and the number of expansion terms is set to $J$ $=100$. (a) Without scaling the elementary wave functions; (b) With the elementary waves scaled on a sphere with radius, $r_{s}=0.25 \mathrm{~m}$.

elementary wave functions, i.e., Fig. 2(a) is based on the elementary waves, $\Psi_{j}$, in Eq. (2), while Fig. 2(b) is based on $\widetilde{\Psi}_{j}$ from Eq. (3). The singular vectors with high index $i$ correspond to the small singular values, and normally in $\mathrm{NAH}$ these singular vectors mainly contain the high spatial frequencies. This is clearly not the case in Fig. 2(a); in fact, the spatial frequency seems to decrease with $i$. In Fig. 2(b) the waves are scaled on a sphere with radius, $r_{s}=0.25 \mathrm{~m}$, and as a result, the left singular vectors with low indices are the least fluctuating ones. If the elementary waves are not scaled, the amplitude of the radial part of the high order expansion terms will be significantly higher than those of low order. For the present setup the SVD decomposes B into basis vectors that behave much like the elementary waves, and the high amplitudes of the radial part will therefore be seen as large singular values associated with the left singular values with high spatial frequencies.

As a consequence of the opposite behavior of the singular vectors, the first basis vectors to be removed from the solution when applying the TSVD in Eq. (7) are those with the lowest spatial frequencies. This is undesirable since these wave components are very important in the solution. Hence, it is reasonable to expect that the TSVD (or Tikhonov) will not benefit the solution at all for this setup.

The particle velocity created by the monopoles will now

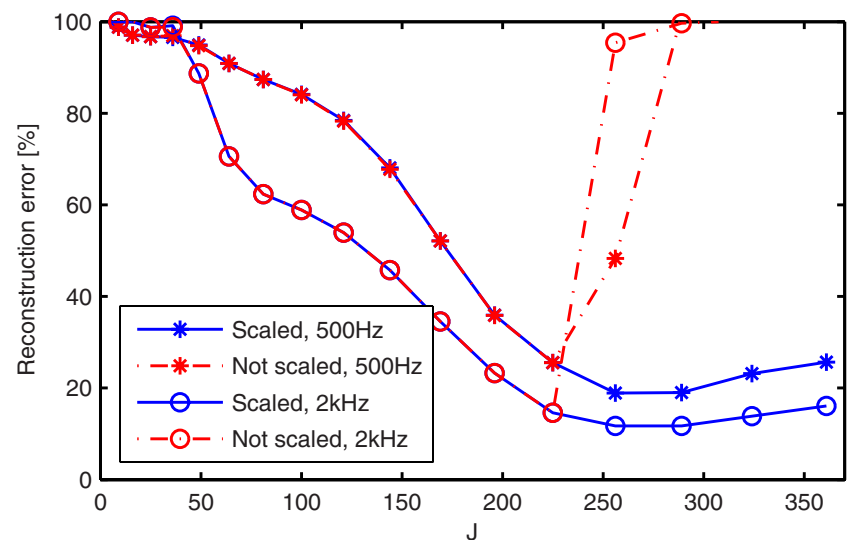

FIG. 3. (Color online) Error in reconstructed velocity in the $15 \times 15$ reconstruction points as a function of the truncation of the elementary waves, $J$. The TSVD is used for all results with the regularization parameter chosen optimally.

be estimated in the normal direction of the reconstruction surface, and Fig. 3 shows the relative errors as a function of the truncation of the spherical wave expansion with and without the wave scaling. The upper limit, $J=(18+1)^{2}=361$, is set so that the elementary waves can be sampled on equator and in the $\theta$-direction with approximately two measurement positions per half-period. The regularization parameter in the TSVD is chosen optimally, i.e., yielding the lowest possible reconstruction error. Up to $J=225$ the optimal results are obtained when no regularization is introduced, which is the reason why the curves coincide up to this truncation number. Above this value the error does not change much for the scaled version since the terms above $J=225$ are effectively removed by the TSVD. For the unscaled waves, however, the SVD cannot be truncated because the low order components are important in the solution.

\section{B. Open measurement surface}

In practice it is often not possible to measure the sound field on a surface that surrounds the source. The next simulation uses the open measurement surface from Fig. 1(d) that covers only a patch on the spherical surface from before. This type of array will be denoted as a patch array. The elementary waves are again scaled on the reconstruction surface $\left(r_{s}=0.25 \mathrm{~m}\right)$.

The left singular vectors for the patch array are shown in Fig. 4. The vectors seem to be ordered in the traditional way with the spatial frequencies increasing with $i$. It should be kept in mind, though, that an elementary wave with high order might not vary much across the small patch, which means that high order elementary waves then behave as singular vectors with low index. As an example, the elementary wave function, $\Psi_{100}$, from Eq. (3) only has approximately one half-period across the patch in the $\theta$-direction and two periods in the $\phi$-direction (not shown). This means that some elementary waves with relatively high order mainly consist of combinations of singular vectors with relatively low index. The singular vectors with low index might even contribute significantly to both a high and a low order elementary wave, respectively. The remains, which cannot be repre- 


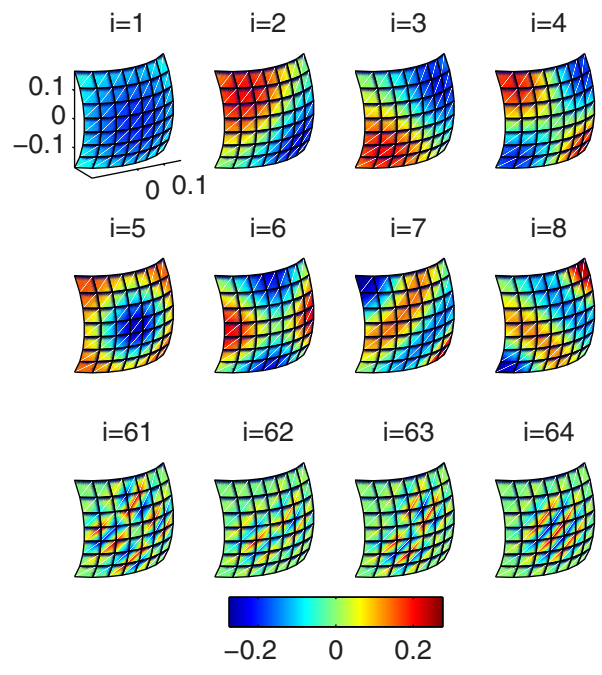

FIG. 4. (Color online) Real values of the left singular vectors, $\mathbf{u}_{i}$, plotted on the open measurement surface. The elementary waves are scaled on $r_{s}$ $=0.25 \mathrm{~m} ; J=100$ and $f=500 \mathrm{~Hz}$.

sented by the first singular vectors, will be seen as the noiselike singular vectors in the bottom row in Fig. 4.

To analyze the inverse problem further, Eq. (6) is multiplied by $\mathbf{v}_{i}^{H}$ yielding

$$
\mathbf{v}_{i}^{H} \mathbf{a}=\frac{1}{\sigma_{i}} \mathbf{u}_{i}^{H} \mathbf{p}, \quad i=1,2, \ldots, I,
$$

where the orthogonality of the right singular vectors, $\mathbf{v}_{i}$, has been used. The inner product, $\mathbf{u}_{i}^{H} \mathbf{p}$, describes the $i$ th left singular vector's contribution to the measured pressure, and the $j$ th element in $\mathbf{v}_{i}$ is multiplied on the amplitude of the $j$ th elementary wave, which means that the magnitude of the $j$ th element in $\mathbf{v}_{i}$ is proportional to the participation factors of the $j$ th elementary wave. In the simple case where all the coefficients are unity, the left-hand side becomes a sum of the elements in $\mathbf{v}_{i}^{H}$, and the element with highest amplitude participates the most to the inner product, $\mathbf{u}_{i}^{H} \mathbf{p}$. Figure 5 shows the modulus of the elements of the matrix, $\mathbf{V}^{H}$ $=\left\{\mathbf{v}_{1}, \mathbf{v}_{2}, \ldots, \mathbf{v}_{I}\right\}^{H}$, where $I=\operatorname{rank}(\mathbf{B})$, when using the sphere array and the patch array, respectively. For the sphere array the high-magnitude elements are mainly located around the diagonal, which means that the elementary waves with low $j$ (the first columns in $\mathbf{V}^{H}$ ) are closely related to the singular vectors with low $i$ (the first rows in $\mathbf{V}^{H}$ ) and vice versa. Notice that the matrix is very well divided in clusters with $2 n+1$ rows and columns, where $n$ is the order of the wave functions belonging to the cluster. For the patch array the concentration of high-magnitude elements is more blurred and the matrix is not dominated around the diagonal. The singular vectors with relatively low index, $i$, involve elementary waves with relatively high index, $j$, and already at $i$ $\approx 15$ all the included terms have come into play. This is in agreement with the arguments given in connection with Fig. 4 regarding the fact that a high order elementary wave does not necessarily vary much across the patch.

Knowing that the singular values decrease when $i$ is increased, it can be concluded from Fig. 5 that for the sphere array, the level of the singular values is directly related to the
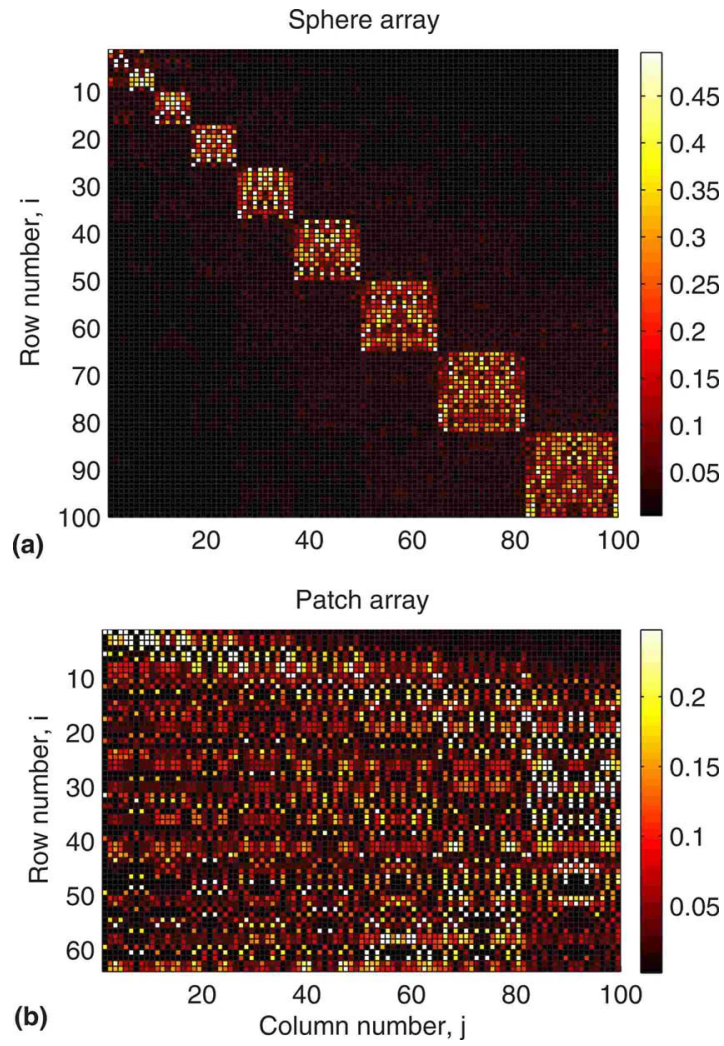

FIG. 5. (Color online) Modulus of the matrix elements in $\mathbf{V}^{H}$ for $f$ $=500 \mathrm{~Hz}$ and $J=100$, (a) with the sphere array, and (b) with the $8 \times 8$ array covering only a part of the spherical measurement surface.

order of the elementary waves. This is not the case for the patch array, which is recognized by the fact that $\mathbf{V}^{H}$ is not dominated around its diagonal.

Figure 6 shows the singular values for both the patch array and the sphere array. The plot shows that $\sigma_{i}$ drops faster for the patch array, which can again be explained by the fact that for the patch array the elementary waves' variation across the array area consists of combinations of the singular vectors with low index $i$, and those with high index in Fig. 4 make up a small contribution to the elementary waves, resulting in small singular values. That is, there exists some level of linear dependence between the elementary

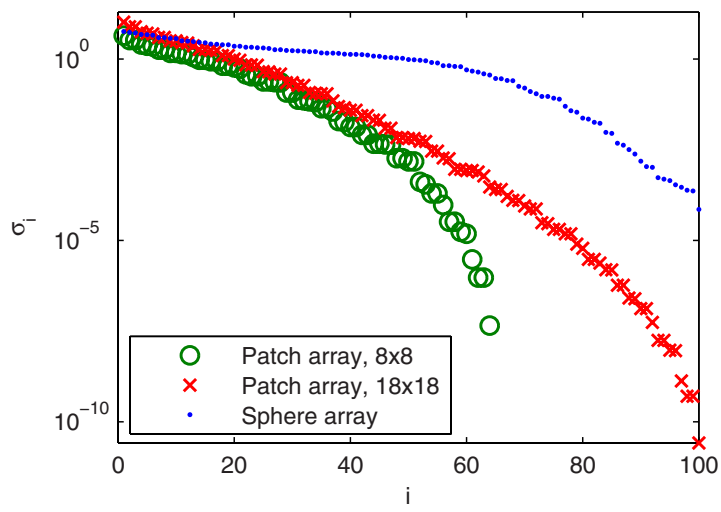

FIG. 6. (Color online) Singular values for an open (patch) array and a closed (sphere) array. Two patch arrays are used: One with $8 \times 8$ and one with $18 \times 18$ measurement positions; the two patch arrays cover the same area on the measurement sphere. The elementary waves are scaled on $r_{s}=0.25 \mathrm{~m}$; $J=100$ and $f=500 \mathrm{~Hz}$. 
waves seen across the small patch area and this linear dependence translates into a faster decay of $\sigma_{i}$. For the sphere array the elementary waves behave very differently seen over the closed measurement surface. In fact, the elementary waves are even orthogonal when considering the entire sphere, and therefore the wave functions are "more linearly independent" than for the patch array, which results in a slower decay of the singular values. Figure 6 also shows the singular values corresponding to a patch array with $18 \times 18$ measurement positions, covering the same area as the $8 \times 8$ array. This array is included in the plot to show that the conclusion also holds for a patch array with roughly the same number of microphones as the sphere array. It should be emphasized that the measurement surface need not be of spherical shape to have a low condition number, i.e., a small ratio between the largest and the smallest singular value. For instance, spot checks with a box-shaped measurement surface also resulted in matrix with a high concentration around the diagonal as the one in Fig. 5 and small condition number (not shown).

\section{Comparison of the regularization approaches}

In this section, the SOSWE results are compared with and without the use of the TSVD, respectively. The test case from Fig. 1 is used again, and the resulting reconstruction errors are shown in Fig. 7 for the $8 \times 8$ patch array and for the sphere array. From Fig. 7(a) it is seen that up to $J=225$ the TSVD is unnecessary when using the sphere array. The condition number increases with $J$ due to the strong decay of the elementary waves with highest order, and at $J>225$ the condition number becomes so large that the error of the unregularized solution starts to increase. Hence, for this array configuration the TSVD can be omitted if the truncation number, $J$, is chosen optimally. However, this requires an automated method for the selection of $J$, and a wrong selection will result in large errors. When introducing the TSVD, the error does not increase much at high values of $J$ because the part of the solution stemming from the small singular values is removed. Therefore, when the TSVD is used, the method is not very sensitive to the choice of $J$, as long as it is chosen large enough.

As mentioned, the TSVD is superfluous at low values of $J$ for the sphere array, but as seen in Fig. 7(b), this is not the case for the patch array. The noise in $\mathbf{p}$ will in general have components as those in the bottom row of Fig. 4 and the associated small singular values result in an unwanted amplification of these components during the reconstruction. Therefore, the additional regularization is required for most of the values of $J$ in Fig. 7(b). These plots clearly show that there is a risk of getting high errors if additional regularization is not implemented in the SOSWE; at least if the measurement surface covers only a patch on an otherwise closed surface.

\section{Nonspherical source shape}

Very often in applied acoustics the vibrator of interest has a nonspherical shape. The theory behind SOSWE dictates that the source must be of spherical geometry in order
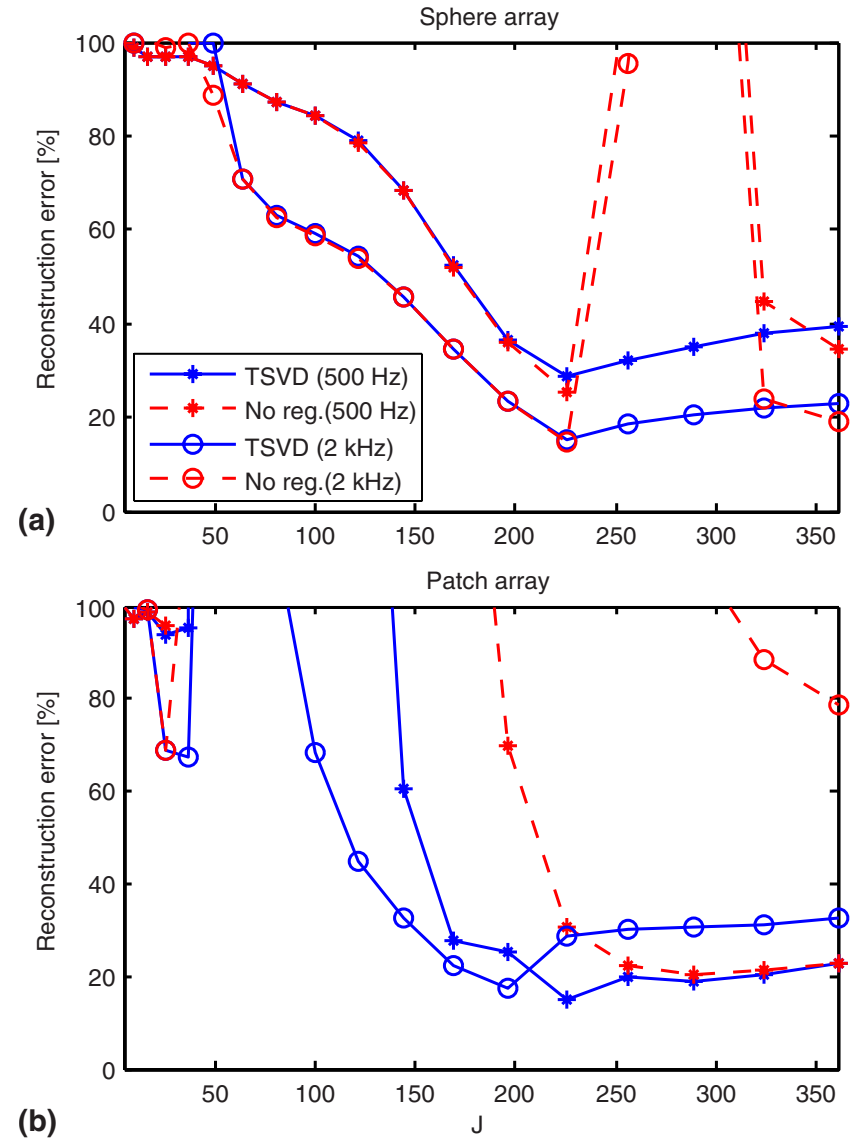

FIG. 7. (Color online) Error in the velocity as a function of the number of elementary waves included with (a) patch array, and (b) sphere array. The dashed lines are based on Eq. (5), i.e., without the TSVD as additional regularization, and the solid lines are based the TSVD solution in Eq. (7) with GCV as parameter choice method. The waves are scaled at $r_{s}$ $=0.25 \mathrm{~m}$.

to reconstruct the sound field on its surface, but as mentioned, earlier results have shown reasonable accuracy for the method when applied on nonspherical source shapes as well. This section investigates the reconstruction errors using a planar vibrator.

The simulated source object is a $0.08 \times 0.36 \times 0.21 \mathrm{~m}^{3}$ rectangular box with five rigid sides and the sixth side being a $0.36 \times 0.21 \mathrm{~m}^{2}$ simply supported steel plate. The plate is point excited near a corner, and the radiated sound field is calculated using the boundary element method. The sound pressure is computed at $8 \times 8$ field positions situated $3 \mathrm{~cm}$ from the plate (see Fig. 8). Microphone mismatch is again added to the sound pressure, and the result is used as input to SOSWE to find a set of expansion coefficients. Finally, the particle velocity is estimated at $15 \times 15$ points on the plate directly under the array. The waves are scaled on a sphere with a radius equal to the average distance between the expansion origin and the reconstruction points.

Two thicknesses have been used for the plate, 5 and $1.5 \mathrm{~mm}$. In general, the dominating modes in the steel plate at a given excitation frequency will have a shorter spatial wavelength for the thin plate. This means that the radiated sound field is more evanescent for the $1.5 \mathrm{~mm}$ plate than for the $5 \mathrm{~mm}$ plate. The resulting reconstruction errors are shown in Fig. 9 for the $5 \mathrm{~mm}$ plate at two different frequen- 


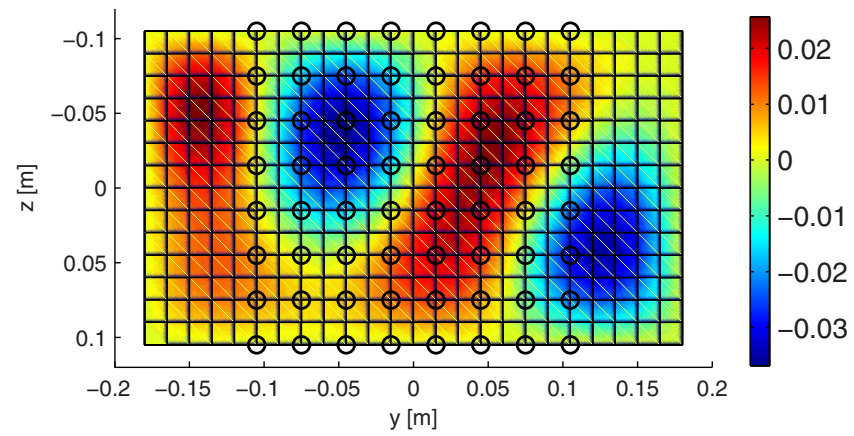

FIG. 8. (Color online) Imaginary part (the real part i zero) of the normal velocity in $\mathrm{m} / \mathrm{s}$ of a simply supported steel plate (in a rigid rectangular box). The circles indicate $8 \times 8$ microphone positions placed $3 \mathrm{~cm}$ from the plate. The displayed vibration pattern is for a $5 \mathrm{~mm}$ plate point excited at $500 \mathrm{~Hz}$.

cies. Each curve on the plots corresponds to a new distance between the expansion origin and the measurement plane, $a$. The results show that there is an interrelation between the optimal number of elementary waves and the distance to the expansion origin, and the optimal combination of $a$ and $J$ clearly depends on the frequency. In general, the further away the origin is, the higher is the optimal number of functions. This can be explained by the fact that the elementary waves propagate for $k a>n$, which means that the order, $n$, must be high to represent the evanescent behavior of the field when $a$ is large.

From Fig. 9 it seems that good results can be achieved if

(a)
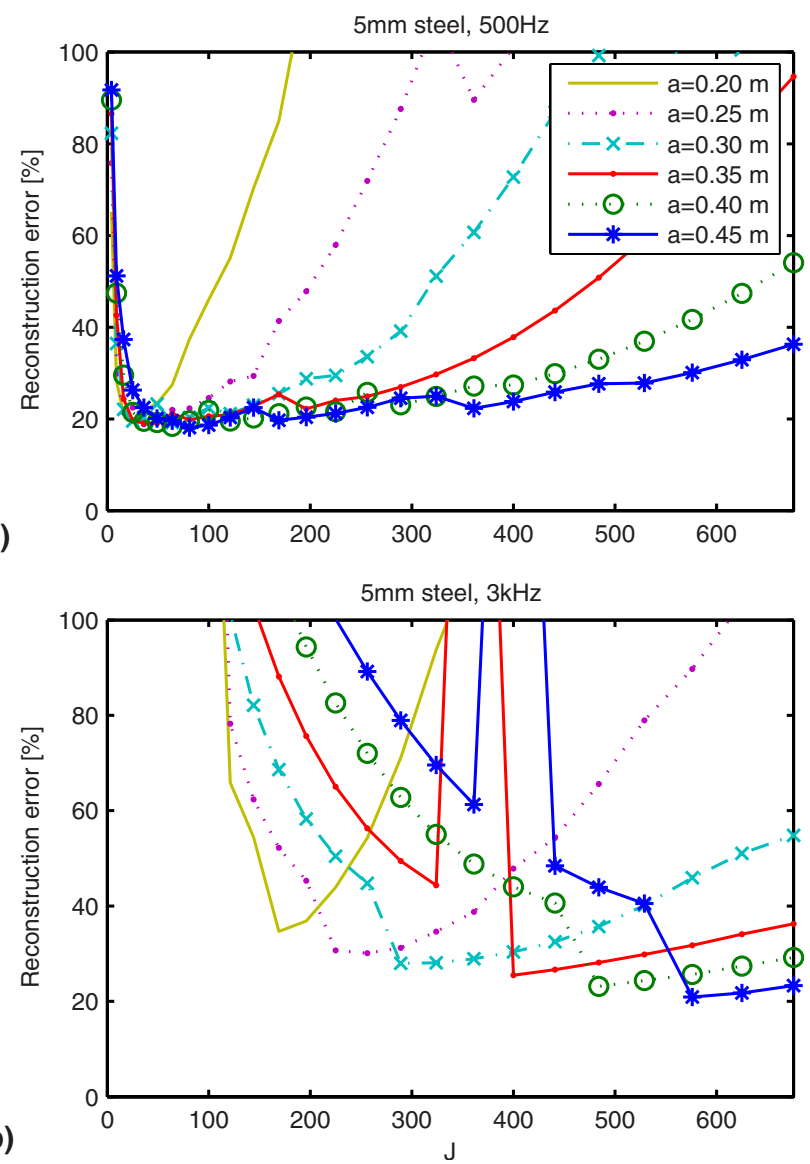

FIG. 9. (Color online) Relative error in the reconstucted particle velocity on the vibrating $5 \mathrm{~mm}$ steel plate for different distances, $a$, to the expansion origin. GCV is used to find a regularization parameter for the TSVD.
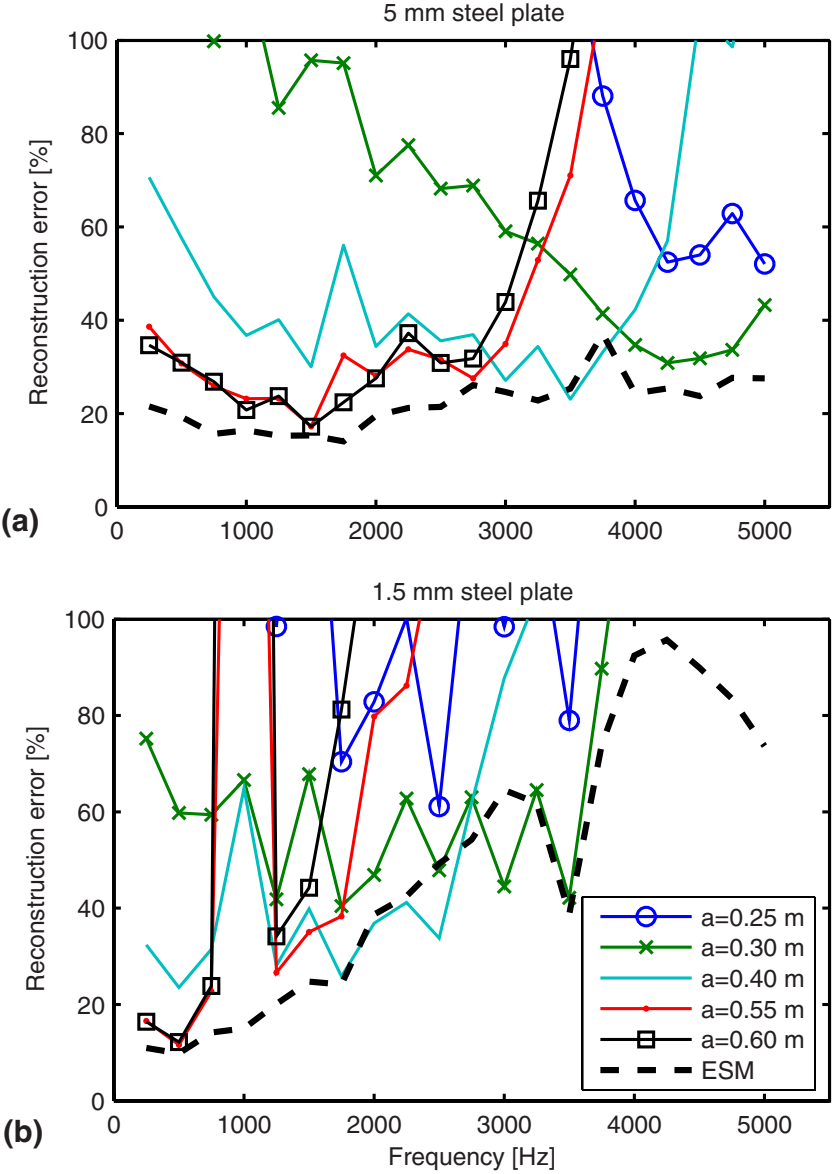

FIG. 10. (Color online) Reconstruction error as a function of frequency; (a) $5 \mathrm{~mm}$ steel plate, and (b) $1.5 \mathrm{~mm}$ steel plate. Each curve corresponds to a new distance to the expansion origin, $a$; $J=676$; GCV is used with the TSVD. The ESM results are also shown for the sake of benchmarking.

$J$ is set high and if the origin is adjusted accordingly. Figure 10 shows the error as a function of frequency for the two plates using different positions of the expansion origin with a fixed number of terms, $J=676$. No single value for $a$ benefits the whole frequency range. For the low frequencies the best results are obtained with the origin far from the plate, and for the high frequencies the preferred origin is closer to the plate. For the furthest origins, e.g., $a=0.6 \mathrm{~m}$, better results are achieved if a higher value of $J$ is used (not shown), but the choice for $J$ is a compromise between computational cost and the (assumed) reconstruction accuracy.

The errors are also shown for ESM in Fig. 10 for benchmarking. The equivalent sources (monopoles) are positioned below the plate at a distance equal to the spacing of the reconstruction points $(1.5 \mathrm{~cm})$. There is one monopole below each reconstruction point, and then two additional rims of monopoles at the edges, i.e., $19 \times 19$ in total with a $1.5 \mathrm{~cm}$ spacing. ESM is clearly more robust and accurate than SOSWE, and the position of the monopoles does not have to be varied with the frequency in order to get satisfactory results.

\section{EXPERIMENTAL RESULTS}

Some experiments have been carried out in the anechoic room at the University of Southern Denmark. The source 


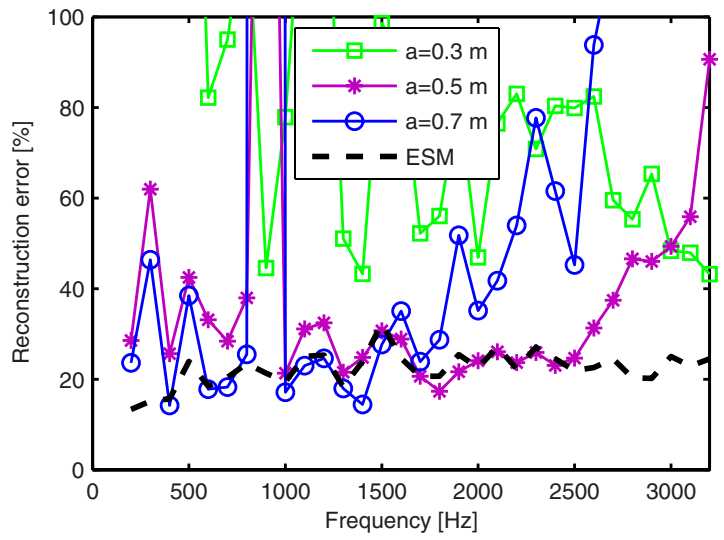

FIG. 11. (Color online) Experiment with a vibrating steel plate. Error in reconstructed velocity using SOSWE with different expansion origins and ESM.

object is a $0.4 \times 0.4 \times 0.5 \mathrm{~m}^{3}$ rectangular box of $19 \mathrm{~mm}$ fiberboard with one side replaced by a $3 \mathrm{~mm}$ steel plate.

The steel plate was excited at a point near its center with broad band noise using a Brüel \& Kjær (B\&K) exciter (type 4809). A stinger was mounted on the exciter, and a force transducer connected the stinger to the plate (using beeswax on the transducer). The pressure was measured with a $\mathrm{B} \& \mathrm{~K}$ two-layer microphone array with $8 \times 81 / 4$ in. microphones (type 4959) in each layer. The microphones constitute a regular grid with $3 \mathrm{~cm}$ spacing, and only the front layer of microphones was used. The array was positioned symmetrically with respect to the steel plate at a standoff distance of $3 \mathrm{~cm}$. A B\&K "PULSE" analyzer with 65 channels was used in all measurements.

The normal velocity of the steel plate was measured with a laser vibrometer (Ometron VH-1000-D) in $16 \times 14$ points covering the entire plate with a spacing of $3 \mathrm{~cm}$ between the points. The force transducer was used as a reference for both pressure and velocity measurements, and to circumvent problems such as drift in the setup, only transfer functions (between force transducer and laser/microphone) were used. Hence all values in the following are given in $(\mathrm{m} / \mathrm{s}) / \mathrm{N}$ instead of $\mathrm{m} / \mathrm{s}$.

The results from the laser are considered as the true velocity, and Eq. (9) is used as an error measure taking into account only the $8 \times 8$ points in front of the array. Since the difference in phase response between the laser and the microphones is unknown, only absolute values are used in the error calculation.

In the results that follow, the truncation of the wave expansion is set to $J=676$, and GCV is used as parameter choice method in the TSVD. The reconstruction errors using SOSWE with different origins are displayed in Fig. 11. As for the simulated steel plate in Sec. III D, the optimal position of the origin is further away from the plate at the low frequencies and closer to the plate for the higher frequencies. The ESM errors are also shown in the plot, and again, this method is as good, or better, than SOSWE. The equivalent sources in ESM are distributed in the same way as in the simulations.

The optimal location of the origin in SOSWE depends strongly on the number of terms included, the frequency, and

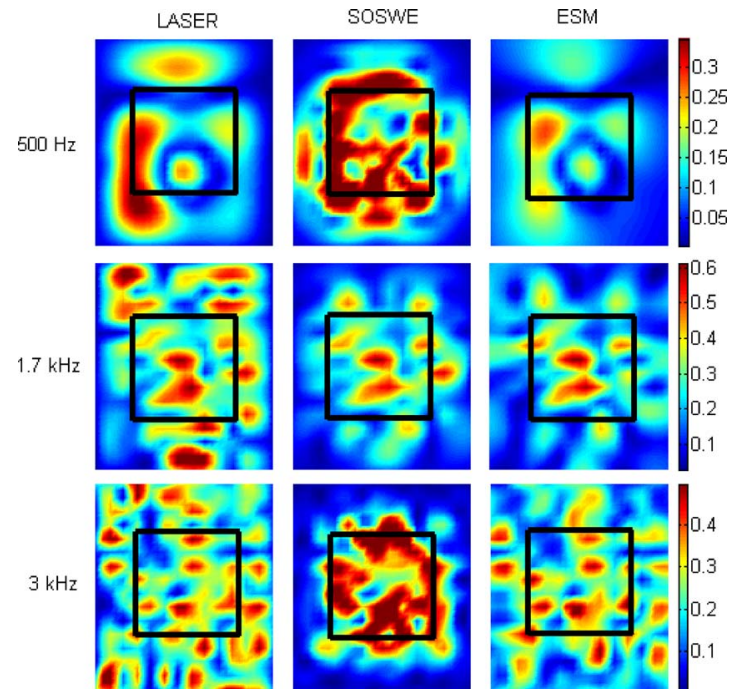

FIG. 12. (Color online) Measured (first column) and estimated (second and third column) normal velocity in $(\mathrm{m} / \mathrm{s}) / \mathrm{N}$ on the steel plate. The black squares indicate the position of the array. The origins in SOSWE are found from the optimization procedure: $a=0.4 \mathrm{~m}$ at $500 \mathrm{~Hz}, a=0.5 \mathrm{~m}$ at $1.7 \mathrm{kHz}$, and $a=0.3 \mathrm{~m}$ at $3 \mathrm{kHz}$

on the source object. In Ref. 6 an optimization procedure is suggested to find an estimate to the optimal value for $J$ with a fixed expansion origin. In the following, a similar procedure will be applied, but with $J$ being fixed and the expansion origin being the optimization parameter. The measurement points are divided into two groups $\left(M_{1}\right.$ and $\left.M_{2}\right)$, and then the pressure data from $M_{1}$ are used as input to the SOSWE algorithm to estimate the pressure in $M_{2}$. This is repeated for different origins, and the origin that yields the lowest error in $M_{2}$ is assumed to be the optimal choice. It should be emphasized, though, that the lowest error in $M_{2}$ does not necessarily correspond to the lowest error on the source surface. Every other measurement position is used for $M_{1}$, and the reconstruction error at the remaining points, $M_{2}$, is found for $a=\{0.25,0.3, \ldots, 0.7\} \mathrm{m}$. That is, there are 32 measurement positions in each group, and no neighboring positions belong to the same group. The resulting reconstruction results are shown in Fig. 12 together with the laser results and the ESM-based reconstruction. The plots show the reconstruction results on the entire steel plate (the error calculations in Fig. 11 are based only on the area below the array). The origins used in SOSWE are selected by the optimization procedure, and the erroneous reconstruction pattern at $f=500 \mathrm{~Hz}$ and $f=3 \mathrm{kHz}$ is due to an inappropriate choice of the origin. That is, the origin that yields the lowest error in $M_{2}$ does not correspond to a well reconstructed pattern on the source surface. ESM predicts the velocity pattern quite well in the region below the array and, in fact, also in the region just outside the measurement area. In general, however, the ESM prediction should only be trusted in the area covered by the array.

\section{CONCLUSION}

The SOSWE approach is sometimes used for NAH purposes, and the truncation of the wave expansion is often used as the only regularization method in order to prevent the 
unwanted amplifications of noise during the reconstruction process. The results in the present paper showed that when the measurement points do not constitute a closed surface surrounding the expansion origin, but only a small patch, the truncation of the wave expansion is in general not sufficient to get accurate results since the condition number can be high even for a relatively low number of expansion terms when using the open array. Therefore, additional regularization such as the TSVD or Tikhonov regularization should be applied in connection with SOSWE methods when using open measurement surfaces. The TSVD can be omitted when using a spherical array surrounding the source, but since the truncation of the expansion then works as the only regularization, the number of terms should be selected carefully.

It has been shown that in order for the additional regularization to work properly the spherical wave functions should be scaled in such a way that the waves with highest order have the lowest amplitude on the measurement surface. This can be done by scaling the functions on the surface of the source. By doing that, the regularization will mainly remove information from the high order terms, leaving the information from the low order terms in the regularized solution.

Acceptable accuracy can be achieved with the method on sources of nonspherical geometry, but the errors depend strongly on the choice of the expansion origin and the number of terms included. However, as shown with simulations and practical measurements, if one of these parameters is fixed the other most be changed with the frequency to get useful results. The optimal value of this parameter is unknown in practice, which leaves a risk of high reconstruction errors. This is a main drawback of the method, and as shown in this paper the ESM, which uses distributed monopoles to represent the sound field, is a more robust and accurate method.

${ }^{1}$ E. G. Williams, Fourier Acoustics: Sound Radiation and Nearfield Acoustical Holography (Academic, London, 1999).

${ }^{2}$ E. G. Williams, N. Valdivia, P. C. Herdic, and J. Klos, "Volumetric acoustic vector intensity imager," J. Acoust. Soc. Am. 120, 1887-1897 (2006).

${ }^{3}$ Z. Wang and S. F. Wu, "Helmholtz equation-least-squares method for reconstructing the acoustic pressure field," J. Acoust. Soc. Am. 102, 20202032 (1997).

${ }^{4} \mathrm{~S}$. F. Wu, "On reconstruction of acoustic pressure fields using the Helmholtz equation least squares method," J. Acoust. Soc. Am. 107, 2511-2522 (2000).

${ }^{5} \mathrm{~N}$. Rayess and S. F. Wu, "Experimental validation of the HELS method for reconstructing acoustic radiation from a complex vibrating structure," J. Acoust. Soc. Am. 107, 2955-2964 (2000).

${ }^{6}$ M. S. Moondra and S. F. Wu, "Visualization of vehicle interior sound fields using nearfield acoustical holography based on Helmholtz-equation least-squares (HELS)," Noise Control Eng. J. 53, 145-154 (2005).

${ }^{7}$ E. G. Williams, "Approaches to patch NAH," Proceedings of Inter-Noise 2003, 2003, pp. 2187-2194.

${ }^{8}$ P. C. Hansen, Rank-Deficient and Discrete Ill-Posed Problems: Numerical Aspects of Linear Inversion (Society for Industrial and Applied Mathematics, Philadelphia, PA, 1997).

${ }^{9}$ J. Prager, "Approximate reconstruction of sound fields close to the source surface using spherical nearfield acoustical holography," J. Acoust. Soc. Am. 122, 2067-2073 (2007).

${ }^{10}$ A. Sarkissian, "Method of superposition applied to patch near-field acoustical holography," J. Acoust. Soc. Am. 118, 671-678 (2005).

${ }^{11} \mathrm{~N}$. Valdivia and E. G. Williams, "Study on the comparison of the methods of equivalent sources and boundary element methods for near-field acoustic holography," J. Acoust. Soc. Am. 120, 3694-3705 (2006).

${ }^{12}$ J. Gomes, "Patch holography using a double layer microphone array," Proceedings of Inter-Noise 2007, in07-122. 